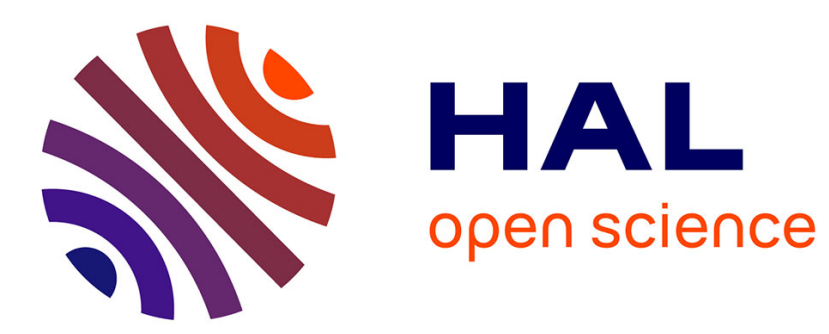

\title{
Linear and nonlinear viscoelasticity of a model polymer melt: Molecular Dynamics and Rouse Modes analysis
}

\author{
Mihail Vladkov, J.-L. Barrat
}

\section{To cite this version:}

Mihail Vladkov, J.-L. Barrat. Linear and nonlinear viscoelasticity of a model polymer melt: Molecular Dynamics and Rouse Modes analysis. Macromolecular Theory and Simulations, 2006, 15, pp.252. 10.1002/mats.200500079 . hal-00005891v2

\section{HAL Id: hal-00005891 \\ https://hal.science/hal-00005891v2}

Submitted on 18 Jan 2006

HAL is a multi-disciplinary open access archive for the deposit and dissemination of scientific research documents, whether they are published or not. The documents may come from teaching and research institutions in France or abroad, or from public or private research centers.
L'archive ouverte pluridisciplinaire HAL, est destinée au dépôt et à la diffusion de documents scientifiques de niveau recherche, publiés ou non, émanant des établissements d'enseignement et de recherche français ou étrangers, des laboratoires publics ou privés. 


\title{
Linear and nonlinear viscoelasticity of a model unentangled polymer melt: Molecular Dynamics and Rouse Modes analysis
}

\author{
Mihail Vladkov*, Jean-Louis Barrat ${ }^{1}$ \\ 1 Laboratoire de Physique de la Matière Condensée \\ et Nanostructures, Batiment Léon Brillouin, \\ 43 Bd du 11 Novembre Université Claude Bernard Lyon 1 \& CNRS \\ 69622 Villeurbanne Cedex, France
}

(Dated: January 18, 2006)

\begin{abstract}
Using molecular dynamics simulations, we determine the linear and nonlinear viscoelastic properties of a model polymer melt in the unentangled regime. Several approaches are compared for the computation of linear moduli and viscosity, including Green-Kubo and non equilibrium molecular dynamics (NEMD). An alternative approach, based on the use of the Rouse modes, is also discussed. This approach could be used to assess local viscoelastic properties in inhomogeneous systems. We also focus on the contributions of different interactions to the viscoelastic moduli and explain the microscopic mechanisms involved in the mechanical response of the melt to external sollicitation.

PACS numbers: $83.10 \mathrm{Mj}, 61.25 \mathrm{Hq}, 83.50 \mathrm{Ax}$
\end{abstract}

\footnotetext{
* To whom correspondence should be addressed. E-mail: mihail.vladkov@lpmcn.univ-lyon1.fr
} 


\section{INTRODUCTION}

The response of polymer melts to mechanical perturbations, involving either oscillatory or steady flow, is of great practical importance, and has been the object of extensive experimental and theoretical studies. [2, 3, 4] This response is well known to be viscoelastic, i.e. the storage and loss moduli exhibit a strong frequency dependence, and nonlinear, with a typical shear thinning behavior for the viscosity.

On the simulation side, the steady state viscosity and shear thinning effects have been studied quite extensively in various configurations for model systems. An extensive review of recent work is given in reference. [5] A spectacular success was the obtention of the Rouse-Reptation (or unentangled-entangled) crossover in the rheological behavior for model polymer melts of the bead spring type.[6] This crossover is obtained for chain lengths of the order of $N=100$ monomers. According to the popular wisdom in the field, melts with $N<100$ should therefore be amenable to a description in terms of the Rouse model, which is considered as a reasonable phenomenological description of unentangled melts.

Investigation of frequency dependent response are much less numerous than for steady state viscosity. In fact we are aware of only one recent study, [8] with objectives quite similar to those of the present article. Our aim is to investigate the mechanical response of model polymer melts submitted to steady or oscillatory shear, in order to obtain a characterization in terms of frequency and amplitude of the solicitation. In view of the numerical cost of such calculations, our study will be limited to short chains, but will explore several values of amplitudes and frequencies, concentrating on relatively low frequencies.

As it turns out, a direct assessment of mechanical properties using non equilibrium molecular dynamics (NEMD) is very costly from a computational viewpoint. Hence it is desirable to explore methods that could provide the same information with a lesser computational effort. We will in particular explore the possibility of obtaining viscoelastic properties directly from a study of Rouse modes. Those, being single chain properties, offer a much better statistical accuracy than the stress itself, which is a global property of the system. 
The systems under study are briefly described in the next section. We then discuss the steady state viscosity, both in the linear and nonlinear regime. We use three different methods to determine the viscosity - NEMD simulations, equilibrium Green-Kubo approach and show how the viscosity can be obtained in a third way from the analysis of equilibrium Rouse modes, provided the contribution from short times is correctly taken into account. We then turn to the study of oscillatory strains by means of NEMD simulations. The conditions for linear response at low frequency are discussed, and the various contributions to stress response - storage and loss - are estimated. Again, the results are compared to an equilibrium analysis based on the Rouse model.

\section{SYSTEM DESCRIPTION AND METHODS}

The chains are modelled by an abstract and generic, though well studied, bead spring model - the rather common "Lennard-Jones + FENE" model. [1] All monomers in the system are interacting through the Lennard-Jones potential:

$$
U_{l j}(r)= \begin{cases}4 \varepsilon\left((\sigma / r)^{12}-(\sigma / r)^{6}\right), & r \leq r_{c} \\ 0, & r>r_{c}\end{cases}
$$

where $r_{c}=2.5 \sigma$. Neighbor monomers in the same chain are linked by the FENE (Finite extension non-linear elastic) potential:

$$
U_{F E N E}(r)=\frac{k}{2} R_{0} \ln \left(1-\left(\frac{r}{R_{0}}\right)^{2}\right), \quad r<R_{0}
$$

where $R_{0}=1.5 \sigma$ and $k=30.0 \varepsilon / \sigma^{2}$. The typical size of the studied systems was of 1280 and 2560 particles (for chain lengths of 10 and 20) in a periodic cubic simulation box. The temperature of the melt was fixed in all simulations at $k_{B} T=1$ and the density was $\rho=0.85$. For the non equilibrium runs we use the Lees Edwards periodic boundary conditions coupled with the SLLOD [9] equations of motion. This algorithm allows us to simulate an unconfined periodic system under shear. The stress tensor in the melt

is calculated as $\sigma_{\alpha \beta}(t)=-\frac{1}{V} \sum r_{i j}^{\alpha} F_{i j}^{\beta}$, where $F_{i j}^{\beta}$ the $\beta$ component of the force between particles $i$ and $j$. The kinetic contribution of the stress $\left(\propto \sum v_{i}^{\alpha} v_{j}^{\beta}\right)$ was also evaluated and found to have a negligible contribution to the stress as expected at high density. The 
simulation time step was taken to be $\delta t=0.005 \tau_{L J}$, where $\tau_{L J}$ is the Lennard-Jones time. All times in the results are in units of LJ time. The simulations were performed using the LAMMPS code. [17]

\section{STEADY STATE SHEAR VISCOSITY}

\section{A. NEMD determination}

The most direct method to obtain shear viscosities is undoubtedly the nonequilibrium approach that generates homogeneous, planar Couette flow using Lees-Edwards boundary conditions and the Sllod algorithm. For a shear rate $\dot{\gamma}$ and a velocity profile $v_{x}(y)=\dot{\gamma} y$, the viscosity is given by

$$
\eta=\frac{\langle\sigma(t)\rangle}{\dot{\gamma}}
$$

Where $\sigma(t)$ is the $x y$ component of the stress tensor in the sample. The viscosity obtained by this method depends on the shear rate, generally decreasing with $\dot{\gamma}$ (shear thinning). An extrapolation is required to estimate the zero shear rate value. Shear thinning generally takes place when $\dot{\gamma}>1 / \tau_{c}$, where $\tau_{c}$ is a characteristic relaxation time of the polymer melt (usually, for unentangled melts, the Rouse time $\tau_{R}$, as can be seen in fig. 1).

Precise measurements for low shear rates are very time consuming as substantial statistics are needed for the accurate determination of $\langle\sigma(t)\rangle$ which has a small value for small shear rates. As the relaxation time of the polymer chains increases rapidly with the chain length $\left(\tau_{c} \propto N^{2}\right)$, for long chains very low shear rates $\dot{\gamma}<1 / \tau_{c}$, should be used to reach the Newtonian plateau in $\eta(\dot{\gamma})$. This is illustrated by the large error bars on the low shear rate side of figure 1. For chains of lengths $N=10$ and $N=20$, our extrapolation at zero shear rate is consistent with the scaling expected for unentangled melts, $\eta \propto N$, and with earlier estimates found in the literature. $\| 8$ 


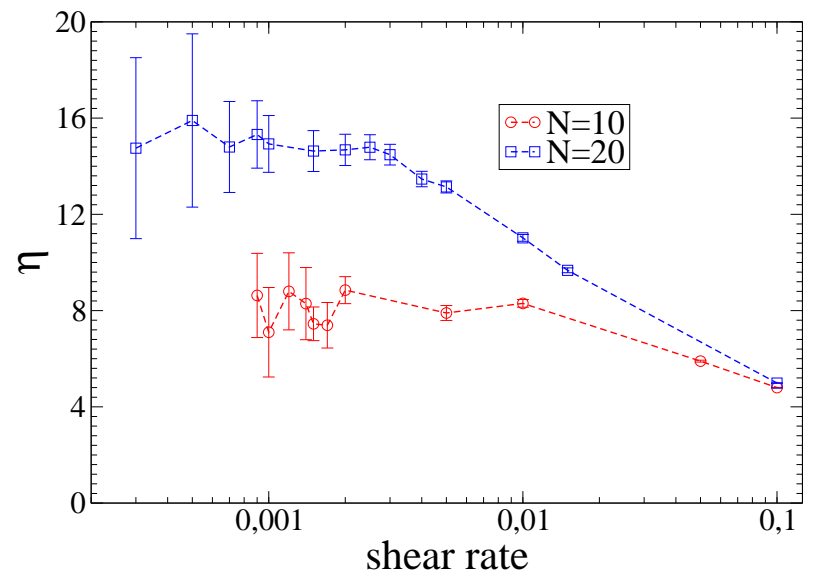

FIG. 1: Shear viscosity as a function of shear rate $\dot{\gamma}$ measured in simulations of planar flow (equation 3). Shear thinning takes place for approximately $\dot{\gamma}>0.01$ for $N=10$ and $\dot{\gamma}>0.0025$ for $N=20$. The estimated Rouse relaxation times for the two chain lengths are respectively about 100 and 400 . Stress was measured every $2 \tau_{L J}$ for a time ranging from $15000 \tau_{L J}$ for high shear rates to $100000 \tau_{L J}$ for low shear rates. Error bars are estimated using block averages for data correlation. 10]

\section{B. Green-Kubo approach}

The problem of extrapolating the shear rate dependent viscosity value does not occur in equilibrium methods. The zero shear rate viscosity is given by the integral of the stress correlation function (Green Kubo relation)

$$
\begin{aligned}
G(t) & =\frac{V}{k_{B} T}\left\langle\sigma_{x y}(t) \sigma_{x y}(0)\right\rangle \\
\eta & =\int_{0}^{\infty} G(t) d t
\end{aligned}
$$

with $T$ and $V$ the melt temperature and volume. Obtaining an accurate value for the slowly decaying stress correlation function involves very long runs in order to have sufficient statistics. Assuming for simplicity that $\sigma(t)$ obeys Gaussian statistics, the standard error in the time correlation function at long times is given by 10

$$
\delta(\langle\sigma(t) \sigma(0)\rangle) \approx \sqrt{\frac{2 \tau_{c}}{t_{\text {run }}}}\left\langle\sigma^{2}\right\rangle
$$


where $\tau_{c}$ is a characteristic correlation time of the data. Given that the stress correlation is a rapidly decaying function (see fig. $1,\left\langle\sigma^{2}\right\rangle \approx 10^{3}\langle\sigma(10) \sigma(0)\rangle$ ), this means that if we want to estimate correlations for a correlation time $\tau_{c} \approx 100$ it would be necessary to run a simulation of $10^{8}$ (units of LJ time) in order to obtain a relative precision of $\sim 10 \%$ for $\langle\sigma(100) \sigma(0)\rangle$. The situation in the polymeric case is particularly unfavorable, since the large viscosity is obtained as the product of a relatively low modulus and a long relaxation time. Hence the stress correlation function is long ranged in time, but has a low amplitude, easily masked by noise associated with the rapid pair-potential part of the stress that does not involve polymer chain relaxation. This estimate is indeed very pessimistic and should be seen as an upper bound of the possible error in the Green Kubo formula. [11] In practice the stress autocorrelation function has several contributions with different weights and correlation times so that the exact error in the Green Kubo integral is difficult to evaluate and depends on the chain length. In our systems of relatively short chains the uncertainty of the Green-Kubo formula concerning viscosity is of the order of $30 \%$ when the integral is carried out to several relaxation times and grows larger as the correlation function is further integrated (see figure 2).

We did not examine the influence of system size on the statistical accuracy of our data. If a larger system should diminish stress fluctuations (by a factor $\propto \sqrt{N}$, where $N$ is the number of particles), a similar effect is expected by longer simulation time (a factor $\propto \sqrt{T})$. As the computational effort for larger system increases roughly as $N \log N$, we do not expect to gain better precision for less computational time this way, so we did not examine the trade off between system size and run time.

We are aware of several determinations of polymer melt viscosity using the Green-Kubo approach. In [12] a good agreement with NEMD results was obtained for short simulation times without discussion on uncertainty. Another calculation was made in [15], where the authors reported the existence of a large noise in the correlation function. This problem was solved by performing running averages to smooth the data, a method that can reduce the noise due to rapid bond vibrations but whose effect on the the intrinsic statistical accuracy of the stress correlation is not obvious. Our data still indicate a large error bar $(30 \%)$, when this error bar is estimated from the three independent components of the 
stress tensor. A viscosity determination based on a Green-Kubo formula in terms of an Einstein relation was presented in [16], unfortunately with relatively little details that would allow us to compare with our results in terms of efficiency and accuracy. It is clear that the Green-Kubo formula remains the only exact way of determining the viscosity from equilibrium simulations, and should be used whenever an "exact" result is required. Although this is feasible with a large computational effort, 16 a detailed report on its accuracy for polymers is still missing (see however [11]). Therefore it seems interesting to discuss an alternative, faster, approach that can be used for example in comparative studies at a moderate computational cost.

\section{Rouse modes}

It is well known that, at least at a qualitative level, the Rouse model can account for the viscoelastic behavior of unentangled polymer melts. Hence, it is tempting to attempt to bypass the difficulty in obtaining the viscoelastic properties stricto-sensu by directly using this model. The large uncertainties discussed above are highly reduced, when we turn to the calculation of single-particle (or single-chain) correlation functions, which are the essential ingredient of the Rouse model. As the final result is an average over $M$ separate functions the standard error at long times should be $\approx\left(2 \tau_{c} / M t_{\text {run }}\right)^{1 / 2}$.

The spirit of the Rouse model consists in assuming that the melt mechanical behavior is dictated by the relaxation of a single polymer chain, the influence of inter chain interactions being limited to the phenomenological friction constant. Consistent with this assumption, the mechanical stress can be calculated from the Rouse modes of the chains [4]

$$
\sigma_{x y}(t)=\frac{\rho k_{B} T}{N} \sum_{p=1}^{N-1} \frac{\left\langle X_{p x}(t) X_{p y}(t)\right\rangle}{\left\langle X_{p x}^{2}\right\rangle_{e q}}
$$

where

$$
X_{p}(t)=\frac{1}{N} \sum_{n=1}^{N} r_{n}(t) \cos \left(\frac{(n-1 / 2) p \pi}{N}\right), p=0, \ldots, N-1
$$

are the Rouse modes, with $N$ the chain length, $\rho$ the monomer number density and $r_{n}(t)$

- the position of the n-th monomer in the chain at the time $t$. Assuming, again in the 
spirit of the Rouse model, independent Rouse modes, the stress correlation from equation (4) can be rewritten as a function of the equilibrium correlation functions for individual Rouse at equilibrium:

$$
\begin{aligned}
G(t) & =\frac{V}{k_{B} T}\left\langle\sigma_{x y}(t) \sigma_{x y}(0)\right\rangle \\
& =\frac{V}{k_{B} T} \frac{1}{T^{s i m}} \int_{0}^{T^{s i m}} d \tau\left(\frac{\rho k_{B} T}{N}\right)^{2} \sum_{p, q} \frac{\left\langle X_{p x}(t+\tau) X_{p y}(t+\tau)\right\rangle}{\left\langle X_{p x}^{2}\right\rangle} \frac{\left\langle X_{q x}(\tau) X_{q y}(\tau)\right\rangle}{\left\langle X_{q x}^{2}\right\rangle} \\
& =\frac{V}{k_{B} T} \frac{1}{T^{s i m}} \int_{0}^{T^{s i m}} d \tau\left(\frac{\rho k_{B} T}{N}\right)^{2} \frac{1}{N_{c}^{2}} \sum_{c, c^{\prime}} \sum_{p, q} \frac{X_{p x}^{c}(t+\tau) X_{p y}^{c}(t+\tau)}{\left\langle X_{p x}^{2}\right\rangle} \frac{X_{q x}^{c^{\prime}}(\tau) X_{q y}^{c^{\prime}}(\tau)}{\left\langle X_{q x}^{2}\right\rangle} \\
& =\frac{1}{T^{s i m}} \int_{0}^{T^{s i m}} d \tau \frac{\rho k_{B} T}{N} \frac{1}{N_{c}} \sum_{c=1}^{N_{c}} \sum_{p=1}^{N-1} \frac{X_{p x}^{c}(t+\tau) X_{p y}^{c}(t+\tau) X_{p x}^{c}(\tau) X_{p y}^{c}(\tau)}{\left\langle X_{p x}^{2}\right\rangle} \\
& =\frac{\rho k_{B} T}{N} \sum_{p=1}^{N-1} \frac{\left\langle X_{p x}(t) X_{p y}(t) X_{p x}(0) X_{p y}(0)\right\rangle}{\left\langle X_{p x}^{2}\right\rangle^{2}}
\end{aligned}
$$

The viscosity is then calculated using equation (5). With this method we observe a substantial gain in precision (see figure 2) and the values obtained are smaller than the non equilibrium estimates, by about the same amount for the two different chain lengths $\left(\eta_{N E M D}-\eta_{e q} \sim 5\right)$.

This result is not surprising given the simplifications of the latter calculation. The interactions between chains in the melt are not taken into account and the Rouse model cannot yield information about stress relaxation on very short "non - polymer" time scales. In order to get a better understanding of the deficiencies in the calculation using the Rouse model, we first compute the individual relaxation times of the modes. The relaxation times, shown in figure 3, are extracted from the exponential decay of these correlation functions. The Rouse scaling $\tau_{p} \propto 1 / p^{2}$ is well obeyed for the first modes. [13] While this is a typical result for the bead-spring model, [13] we note that using more detailed atomistic simulations larger deviations from the Rouse scaling can be observed, especially in higher modes. [14] For both chain lengths, the relaxation time of the fastest mode was found to be $\tau_{N-1} \sim 2$.

It is clear that the Rouse calculation cannot account for the contribution to the viscosity associated with time scales shorter than $\tau_{N-1}$. On such short time scales the Green-Kubo 


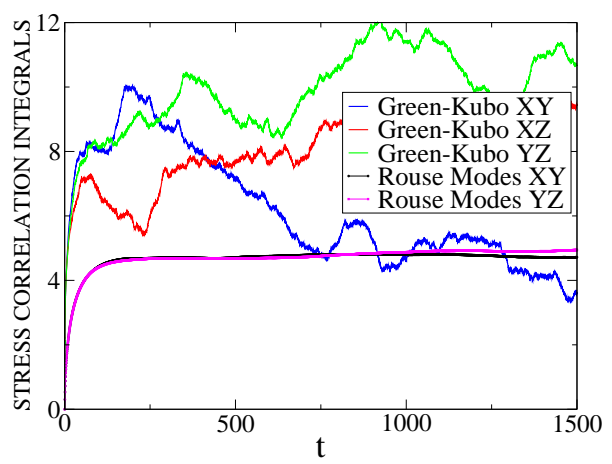

FIG. 2: Integrals of the stress correlation function $G(t)$ for $N=10$ obtained from the global stress (Green-Kubo) and the Rouse modes stress. A distinct plateau that stays stable for very long times $\left(\sim 50 \tau_{R}\right)$ is clearly reached with the Rouse modes formulation. For comparison the Green-Kubo integral value has important fluctuations for times longer than several times the Rouse relaxation time $\tau_{R}$ as predicted by the uncertainty calculation. Global stress was measured every $0.01 \tau_{L J}$ for a time span of $25000 \tau_{L J}$ and $G(t)$ was calculated by taking every point as a starting state and averaging. Such fine sampling is needed to capture the fast oscillations in $G(t)$ shown in fig. 4. Rouse modes were measured every $0.5 \tau_{L J}$ for every chain for a time of $15000 \tau_{L J}$. Than $G^{\text {Rouse }}(t)$ was calculated averaging over starting states and chains.

viscosity integrand can however be obtained with high accuracy, as illustrated in 4 . This stress-stress correlation function first decreases rapidly, and displays a short time damped oscillatory behavior, with a time constant smaller than 0.5. Similar stress oscillations were reported for n-alkanes as well as for a bead-spring melt. [15, 16 In our case, a detailed study of the FENE bonds and Lennard-Jones forces contributions to the stress, shows that these oscillations are due bond vibrations, and that their frequency is close to the intrinsic frequency of the FENE bonds.

Integrating this short time stress correlation function gives the contribution to the melt viscosity of the rapidly decaying part of the global stress, unaccounted for in the Rouse model.

$$
\begin{aligned}
\eta & =\int_{0}^{\infty} G(t) d t \\
& =\int_{0}^{\infty} G^{\text {Rouse }}(t) d t+\int_{0}^{\tau_{N-1}} \frac{V}{k_{B} T}\left\langle\sigma_{\alpha \beta}(t) \sigma_{\alpha \beta}(0)\right\rangle d t
\end{aligned}
$$




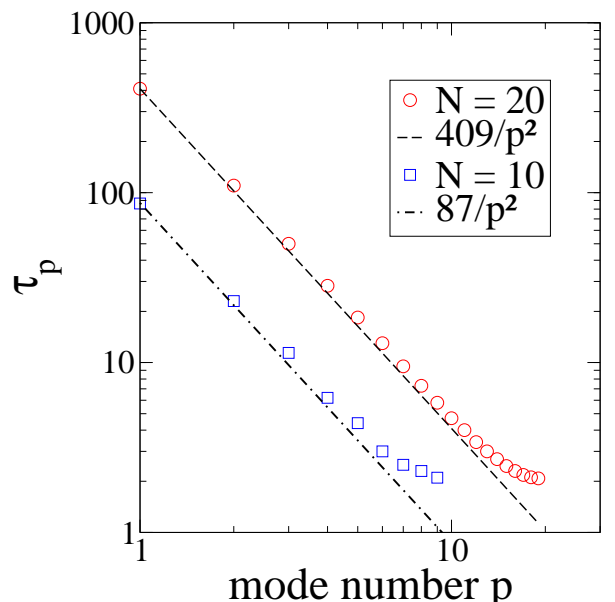

FIG. 3: Relaxation times of the Rouse modes of the chains versus the mode number. Relaxation times were estimated by an exponential fit of the normalized correlation function of the mode, which had in all cases an exponential behavior. Dashed lines show the Rouse theory prediction $\tau_{p} \propto 1 / p^{2}$, followed closely by the first several modes as discussed in 13.

Adding the contribution of the second term of equation 15 to the viscosity from the Rouse model provides a significantly more accurate estimate of the viscosity, compared

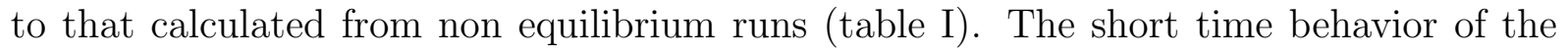
stress correlation function is the same for chain lengths $N=10$ and $N=20$, which is an evidence that this short times contribution is independent of chain length and represents the missing "non-polymeric" part of the mechanical properties of the melt. It provides a contribution to viscosity of the order of the viscosity of a simple LJ fluid at the considered density and temperature.[5]

Being chain length independent, short times stress relaxation represents a part in viscosity that diminishes with increasing chain length, about $40 \%$ for $N=10$ and $\sim 30 \%$ for $N=20$. Still it cannot be neglected for predicting mechanical properties of a melt of unentangled chains. It is equally important for long chains at large shear rates, when the melt viscosity becomes comparable to the one of a simple fluid and can explain deviations from the stress-optic rule. [7]

The presented method provides a way to rapidly obtain an estimate of the viscosity as it requires much less computational time than an accurate Green Kubo measurement. It is 


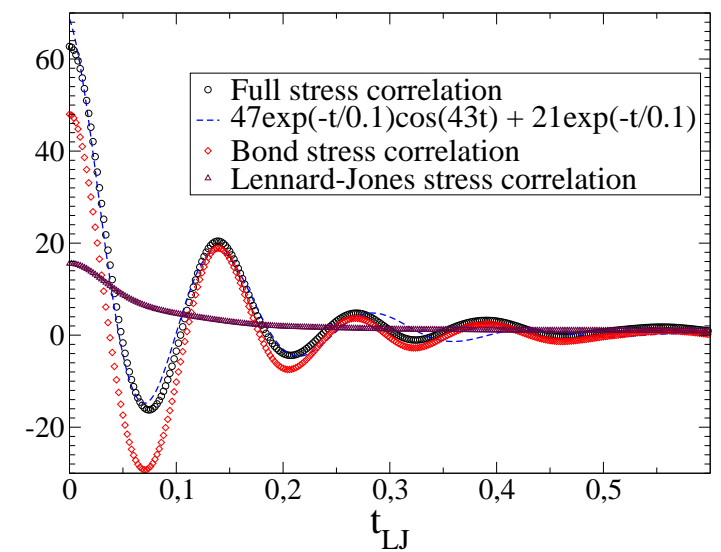

FIG. 4: Global stress correlation functions for short times. Correlations of the pair LennardJones and FENE bond interactions contribution to the global stress are also shown. Dashed line shows a fit of the global stress correlation to indicate the relevant decay time scales and oscillation frequency.

\begin{tabular}{|l||c|c|}
\hline Viscosity & $N=10$ & $N=20$ \\
\hline Green-Kubo estimate & $8 \pm 4$ & $\mathrm{~N} / \mathrm{A}$ \\
Rouse modes value & $4.88 \pm 0.28$ & $10.2 \pm 1.0$ \\
Short times correction & 3.38 & 3.43 \\
NEMD extrapolation & $8.2 \pm 1.3$ & $14.9 \pm 1.9$ \\
Corrected Rouse value & $8.26 \pm 0.28$ & $13.6 \pm 1.0$ \\
\hline
\end{tabular}

TABLE I: Viscosity values from the different methods. Uncertainties in Green-Kubo and Rouse modes $G(t)$ integrals are estimated by evaluating the $\left\langle\sigma_{x y}(t) \sigma_{x y}(0)\right\rangle,\left\langle\sigma_{x z}(t) \sigma_{x z}(0)\right\rangle$ and $\left\langle\sigma_{y z}(t) \sigma_{y z}(0)\right\rangle$ integrals.

still less straightforward and one needs to monitor $N$ (where $N$ is the chain length) Rouse modes for every chain to build the correlation function. The method's main advantage is that, being based on a single chain quantity, it is local in nature and can be used to assess local viscoelastic properties in inhomogeneous systems such as confined melts or melts with filler particles. 


\section{ELASTIC MODULI}

\section{A. Method}

Like the viscosity, elastic moduli can be obtained using either equilibrium or nonequilibrium simulation. If one is interested only in linear response properties, the moduli can be obtained by Fourier transforming the Green-Kubo integrand $G(t)$ (equation 6) in the form

$$
\begin{aligned}
& G^{\prime}(\omega)=\omega \int_{0}^{\infty} d t G(t) \sin \omega t \\
& G^{\prime \prime}(\omega)=\omega \int_{0}^{\infty} d t G(t) \cos \omega t
\end{aligned}
$$

This equilibrium determination, however, suffers from the same drawbacks as the GreenKubo determination of the viscosity, i.e. a long time, small amplitude tail of $G(t)$ has to be known very accurately to obtain reasonable results. That is why we did not show any result concerning elastic moduli issued by a Green-Kubo method. Instead, the only practical way of using equations 16 and 17 is to start from a "model" calculation of $G(t)$, in the sense of the Rouse modeling described in the previous section.

To obtain information beyond the linear regime, the only possibility is to effectively do NEMD and submit the sample to an oscillatory strain, using the standard SLLOD [9] algorithm. The strain is given by

$$
\dot{\gamma}_{0}(t)=\gamma_{0} \omega \sin \omega t \Rightarrow \gamma_{0}(t)=-\gamma_{0} \cos \omega t
$$

And we define the response of the system by the usual formulae for the stress $\sigma(t)$

$$
\sigma(t)=\int_{-\infty}^{t} G\left(t-t^{\prime} ; \gamma_{0}, \omega\right) \dot{\gamma}_{0}\left(t^{\prime}\right) d t^{\prime}
$$

A dependence on the shear amplitude and frequency is indicated in the response function $G$, to recall the possible existence of nonlinear effects. The frequency dependent moduli are defined from the Fourier component of $\sigma(t)$ at the imposed frequency $\omega$

$$
\sigma(t)=\gamma_{0}\left(G^{\prime}\left(\omega, \gamma_{0}\right) \sin \omega t+G^{\prime \prime}\left(\omega, \gamma_{0}\right) \cos \omega t\right)+\text { harmonics at } 2 \omega, 3 \omega . .
$$


The moduli are formally given by the Fourier transforms of the response function

$$
\begin{aligned}
G^{\prime}\left(\omega ; \gamma_{0}\right) & =\omega \int_{0}^{\infty} d t G\left(t ; \gamma_{0}, \omega\right) \sin \omega t \\
G^{\prime \prime}\left(\omega ; \gamma_{0}\right) & =\omega \int_{0}^{\infty} d t G\left(t ; \gamma_{0}, \omega\right) \cos \omega t
\end{aligned}
$$

In practice, $G^{\prime}$ (resp. $G^{\prime \prime}$ ) is extracted from the time series for the stress by multiplying the signal by $\cos (\omega t)($ resp. $\sin (\omega t))$, i.e.

$$
G^{\prime}\left(\omega, \gamma_{0}\right) \int_{0}^{T_{r}} d t \cos ^{2} \omega t=-\frac{1}{\gamma_{0}} \int_{0}^{T_{r}} d t \sigma(t) \cos \omega t+G^{\prime \prime}(\omega) \int_{0}^{T_{r}} d t \cos \omega t \sin \omega t
$$

with $T_{r}$ the length of the simulation run. Thus we obtain the storage and loss moduli as a function of stress :

$$
\begin{aligned}
G^{\prime}\left(\omega, \gamma_{0}\right) & =\frac{2}{T_{r}+\frac{\sin }{2 \omega T_{r}}}\left(-\frac{1}{\gamma_{0}} \int_{0}^{T_{r}} d t \sigma(t) \cos \omega t+G^{\prime \prime}\left(\omega ; \gamma_{0}\right) \frac{\sin ^{2} \omega T_{r}}{2 \omega}\right) \\
G^{\prime \prime}(\omega) & =\frac{2}{T_{r}-\frac{\sin 2 \omega T_{r}}{2 \omega}}\left(\frac{1}{\gamma_{0}} \int_{0}^{T_{r}} d t \sigma(t) \sin \omega t+G^{\prime}\left(\omega ; \gamma_{0}\right) \frac{\sin ^{2} \omega T_{r}}{2 \omega}\right)
\end{aligned}
$$

In the above formulae potential harmonic terms were ignored for simplicity, their contribution being of order $1 / T_{r}$. In the limit $T_{r} \gg 1 / \omega$ one has simply

$$
\begin{aligned}
G^{\prime}\left(\omega ; \gamma_{0}\right) & =-\frac{2}{T_{r} \gamma_{0}} \int_{0}^{T} d t \sigma(t) \cos \omega t \\
G^{\prime \prime}\left(\omega ; \gamma_{0}\right) & =\frac{2}{T_{r} \gamma_{0}} \int_{0}^{T} d t \sigma(t) \sin \omega t
\end{aligned}
$$

In order to elucidate the role of the different interactions for the elastic moduli we focus on their respective contribution. The stress can very generally be separated into an intramolecular stress component associated with FENE bonds and intra chain LennardJones forces, and a intermolecular stress component associated with inter-chain LennardJones interactions. In the following the moduli issued from NEMD simulations will be discussed in terms of these two separate intra and inter molecular contributions. During a NEMD run the full stress in the system as well as its inter and intra molecular components are stored and then elastic moduli are calculated as described above. 


\section{B. Results}

\section{NEMD Results}

The first question that we investigated is the extent of the linear regime, in terms of the strain amplitude. Nonlinear effects can in principle be detected by a dependence of $G(\omega)$ on $\gamma_{0}$, or by the presence of higher harmonics in the stress signal.

There is a clear softening of the response at frequency $\omega$ as amplitude is increased. This softening is obtained above a value of the strain rate $\gamma_{0} \omega$ of the order of $1 / \tau_{R}$ at low frequencies, as illustrated in figure 5. The situation is very similar to the shear thinning behavior of the viscosity, namely the relevant parameter is the shear rate rather than the strain amplitude or frequency.

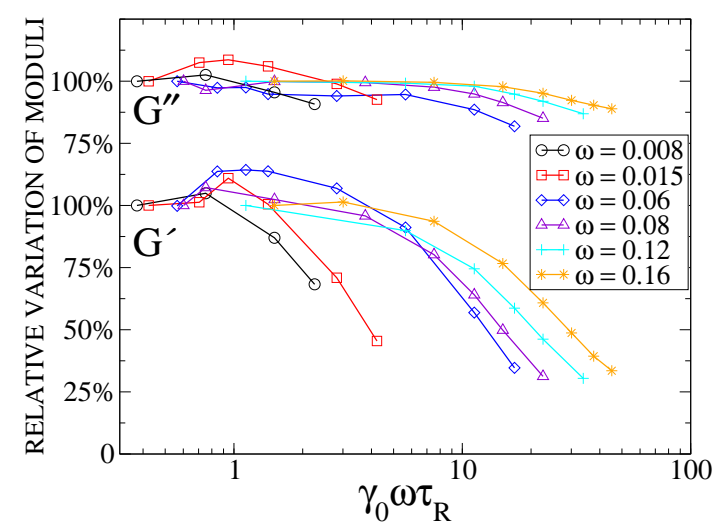

FIG. 5: Dependence of elastic moduli on strain rate amplitude $\gamma_{0} \omega$ for $N=10$. Elastic moduli are normalized by their lowest shear rate amplitude value. For all frequencies the linear regime extends at least to $\gamma_{0} \omega \approx 1 / \tau_{R}$, for $\omega \gg 1 / \tau_{R}$ the linear behavior breaks down for $\gamma_{0} \omega \propto \omega$. The same behavior was measured for $N=20$ (data not shown)

For frequencies $\omega \gg 1 / \tau_{R}$, the softening is observed for values of $\gamma_{0} \omega$ significantly larger than $1 / \tau_{R}$. The behavior of chains in this frequency range is further discussed below. Harmonics were detected in the time series for $\sigma(t)$ at high values of the strain amplitude $\gamma_{0}>2.5$ at a frequency of $3 \omega$, where $\omega$ is the solicitation frequency, for all frequencies. For symmetry reasons the stress must be an odd function of the strain, so that the response function $G$ is an even function of $\gamma$. Hence harmonic contributions are observed only 
for odd multiples of the solicitation frequency. The amplitudes of the harmonic terms in the power spectrum are small, about $8 \%$ and $3 \%$ of the $\omega$ peak for respectively $G^{\prime}(\omega)$ and $G^{\prime \prime}(\omega)$. In the molecular stress harmonics are more visible with about $17 \%$ and $5 \%$ for the storage and loss moduli, respectively. We have not been able to distinguish harmonics for strain amplitudes $\gamma_{0}$ below 2.5. The observation of harmonics can thus be attributed to physical extension of the chains in which the non linear terms in the interaction potentials become inevitably important. Given this preliminary investigation of the non linear regime, we choose first to explore the linear response and place ourselves at shear rates $\gamma_{0} \omega<1 / \tau_{R}$.

Figures 6 and 7 display the frequency dependence of the elastic moduli. As discussed above, the important uncertainty in $G(t)$ obtained from equilibrium calculations imposes the use of non equilibrium methods.

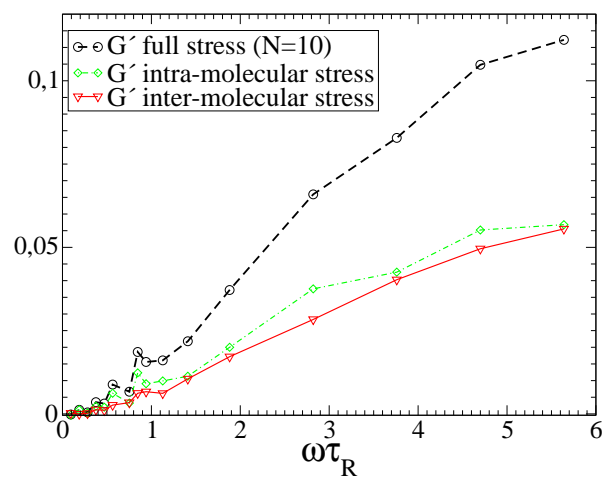

FIG. 6: $G^{\prime}(\omega)$ for $N=10$, measured by NEMD. Contributions from the intra-molecular and inter-molecular forces are shown. Intra molecular forces are important for stress storage up to high frequencies. As $\omega>1 / \tau_{p}$, the modes $X_{i}, i<p$ are "frozen" and behave like stiff springs and store stress efficiently, so that the intra-molecular component of $G^{\prime}(\omega)$ grows with frequency.

The contributions of the different interactions to the elastic moduli (calculated by eqn. (26) and (27)) can be examined by measuring the different contributions to the global stress. The loss modulus, which is a measure of stress dissipation in the melt has several contributions depending on the time scale (fig. 7): at short times stress is relaxed through the pair interactions between monomers, in a "liquid like" manner. At longer times, relaxation of chain fragments of length $N_{p}=1,2 \ldots N$, take place on increasingly 


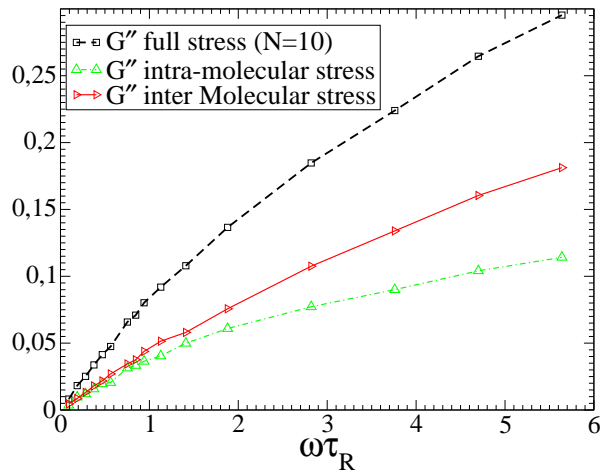

FIG. 7: $G^{\prime \prime}(\omega)$ for $N=10$, measured by NEMD. Contributions to the moduli from the intramolecular and inter-molecular stress components are also shown. For $\omega>1 / \tau_{R}$ the intermolecular contribution to the loss modulus grows larger than the molecular component and the system crosses over to a liquid-like regime.

larger time scales. Knowing that a mode $p$ can be viewed as the relaxation of a subchain of $N_{p}=N / p$ monomers, for a given mode $p$ relaxing over $\tau_{p}$, if the frequency is such that $\omega>1 / \tau_{p}$, the mode cannot relax over one oscillation and does not take part in the stress relaxation, meaning that stress is relaxed on scales smaller than $N / p$ monomers. This leads to the Rouse model prediction that, if chain relaxation was the only process in the melt, the loss modulus had to decrease at high frequencies. This decrease was not observed in our model melt (fig. 7), due to non-polymer relaxation. As non-polymer relaxation we refer to the relaxation of stress occuring on a time scale smaller than the relaxation of the fastest Rouse mode and independent of chain length. Results show that at high frequencies the behavior of the loss modulus is dictated by inter molecular interactions, the inter molecular stress component is dominant. There is a crossover from polymer melt behavior where stress dissipation is carried out mainly by chain relaxation to a behavior of a liquid of interacting chains that do not have time to significantly change their conformation over one period. In this regime there is no stress dissipation due to internal polymer chain relaxation and the increase of the loss modulus with frequency is entirely due to Lennard Jones pair interactions on very short time scales. The situation is somewhat different for the storage modulus, where simulations show that internal polymer chain interactions and chain modes are important for the elastic response of the melt up 
to high frequencies (fig. 6). For a simple liquid the value of $G^{\prime}(\omega)$ is very small in the considered frequency range, so it is not surprising that its value for the melt is due to the chains. The stress storage thus takes place to a large extent in the slowly varying chain conformations. Over a large frequency range the slow vibration modes act as an energy reservoir and the higher the frequency, the more the chains remain rigid at the time scale of a single period and thus cause the increase in the storage modulus with frequency. The melt exhibits an elastic behavior due to the chains that grows stronger with frequency, in fact the mechanism exposed for the loss modulus can be applied the other way around for $G^{\prime}(\omega)$. A given mode $p$ goes rigid as $\omega>1 / \tau_{p}$ and thus stores stress (rigid behavior) instead of relaxing it (liquid behavior). As $\omega>1 / \tau_{p}$, the modes $X_{i}, i<p$ are "frozen" and take an important part in energy storage. For very high frequencies $\omega>1 / \tau_{N-1}$ the chain behaves more like a spring than a flexible polymer.

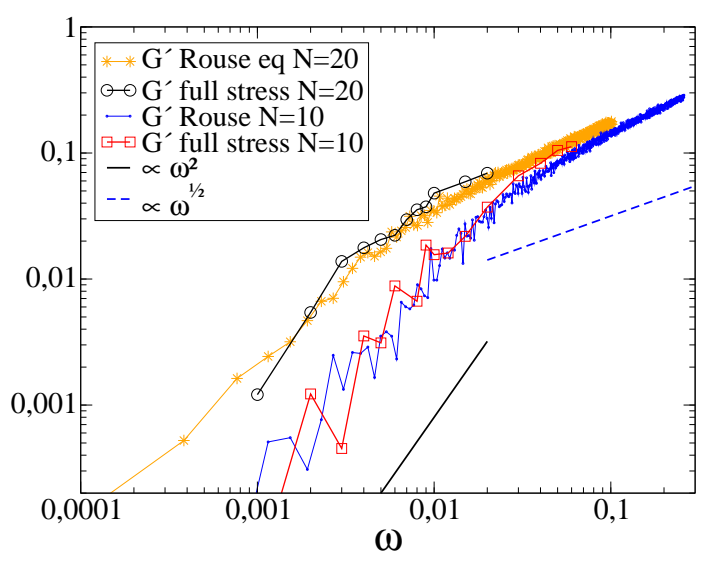

FIG. 8: Storage modulus from NEMD and equilibrium Rouse modes calculation for chains of length $N=10$ and $N=20$. For high frequencies the response of both systems: $N=10$ and $N=20$, becomes identical.

\section{Calculation from Rouse modes and comparison to NEMD data}

An analytical calculation of $G^{\prime}(\omega)$ and $G^{\prime \prime}(\omega)$ can be done using the Rouse model [2, 41. For the bead-spring polymer melt studied here these functions can be estimated using $G^{\text {Rouse }}(t)$ (eqn. 13) via the integrals in equations (21) and (22). Given that, as 


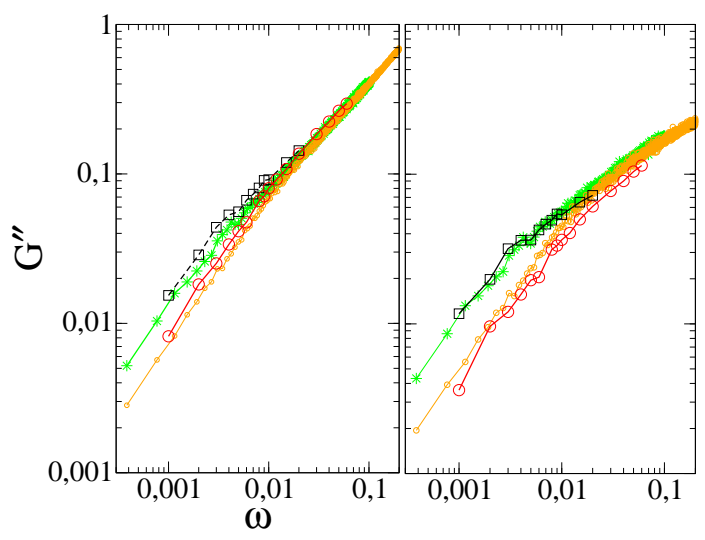

FIG. 9: Elastic loss modulus from NEMD full stress (left, $N=20$ - squares, $N=10$ - circles), NEMD intra molecular stress (right, $N=20$ - squares, $N=10$ - circles), corrected equilibrium Rouse modes calculation (eqn. 32) (left, $N=20$ - stars, $N=10$ - small circles) and Rouse calculation without short times corrections (right, $N=20$ - stars, $N=10$ - small circles). The uncorrected Rouse calculation fits well the intra molecular moduli component. Stress relaxation for high frequencies is identical for the two systems, dictated by short subchain and inter molecular forces relaxation on short time scales as discussed.

discussed above, the stress storage is dictated by slow, "frozen" chain vibration modes for the whole frequency range, the melt elastic response is well reproduced by this calculation (see fig. 8). As shown in fig. 9, the Rouse approach leads to an underestimate of the loss modulus, especially at high frequencies $\left(\omega>1 / \tau_{R}\right)$. The increase in $G^{\prime \prime}(\omega)$ for high frequencies is due to short time non-polymeric stress relaxation discussed in the previous section. As discussed in the first part concerning viscosity, this contribution cannot be predicted by $G^{\text {Rouse }}(t)$ that takes into account only chain vibration modes. As no inter molecular forces whatsoever can be taken into account by the single chain Rouse model, $G^{\text {Rouse }}(t)$ provides a good approximation of the loss modulus calculated from the intra molecular stress component and shows a discrepancy with the full stress loss modulus that grows with frequency. In order to obtain an equilibrium single chain estimate of the mechanical behavior of the melt for all frequencies, we estimate the short time corrections to $G^{\text {Rouse }}(t)$ in a manner similar to the one used for viscosity. We use the fit of the short times stress correlation function (fig. (4) to calculate the short times correction to the 
elastic moduli in the frequency domain by equations (21) and (22). Writing the short time stress correlation in the form

$$
G^{f a s t}(t)=A e^{-t / \tau_{1}} \cos \Omega t+B e^{-t / \tau_{2}}
$$

leads to

$$
\begin{aligned}
G^{\prime}(\omega) & =\omega \int_{0}^{\infty} G^{\text {Rouse }}(t) \sin \omega t d t \\
& +\frac{A}{2}\left(\frac{\omega(\omega+\Omega) \tau_{1}^{2}}{1+(\omega+\Omega)^{2} \tau_{1}^{2}}+\frac{\omega(\omega-\Omega) \tau_{1}^{2}}{1+(\omega-\Omega)^{2} \tau_{1}^{2}}\right)+B \frac{\omega^{2} \tau_{2}^{2}}{1+\omega^{2} \tau_{2}^{2}} \\
G^{\prime \prime}(\omega) & =\omega \int_{0}^{\infty} G^{\text {Rouse }}(t) \cos \omega t d t \\
& +\frac{\omega \tau_{1} A}{2}\left(\frac{1}{1+(\omega+\Omega)^{2} \tau_{1}^{2}}+\frac{1}{1+(\omega-\Omega)^{2} \tau_{1}^{2}}\right)+B \frac{\omega \tau_{2}}{1+\omega^{2} \tau_{2}^{2}}
\end{aligned}
$$

where we determine the parameters $A, B, \Omega, \tau_{1}$ and $\tau_{2}$ from the stress correlation function $\left(A=45, \Omega=43, \tau_{1}=\tau_{2}=0.1\right.$ and $B=21$ for both $N=10$ and $\left.N=20\right)$. Adding these terms to the equilibrium Rouse modes loss modulus gives a much better estimate of $G^{\prime \prime}(\omega)$, producing the curves referred to as corrected Rouse (fig. 9, corrected Rouse). The correction concerning $G^{\prime}(\omega)$ is negligible for the frequency range studied here and the corrected curve falls on top of the original Rouse curve. This is the expected result knowing that, as already mentioned, the liquid like interactions that dominate at short times participate in stress storage only at very high frequencies. We find the expected linear dependence of $G^{\prime \prime}(\omega)$ for $\omega<1 / \tau_{R}$ (fig. 11), the slope being within error bars the value of the viscosity estimated by planar Couette flow simulations. The storage modulus has, as expected, $\sim \omega^{2}$ behavior at low frequencies $\left(\omega<1 / \tau_{R}\right)$ (fig. 10). The Rouse theory predicts a cross over towards $\mathrm{a} \propto \sqrt{\omega}$ behavior for higher frequencies. [4] Our simulations show that $G^{\prime}(\omega)$, estimated by NEMD and Rouse modes measurements, grows slightly faster than $\sqrt{\omega}$ at high frequencies (fig. 10). We can relate this to the discrepancy between theoretical and measured modes relaxation times and argue that the "mean field" presence of multiple chains in our vibration modes determination promotes more efficient stress storage in the melt at high frequencies. Simulations show that $G^{\prime \prime}(\omega)$ does not follow the $\sqrt{\omega}$ behavior at high frequencies either. The loss modulus calculated from the intra molecular stress component, as well as the direct Rouse determination 
follow closely the square root behavior, but the contribution of "non polymer" short time scale inter chain forces change this behavior to almost completely mask the "polymeric" cross over.

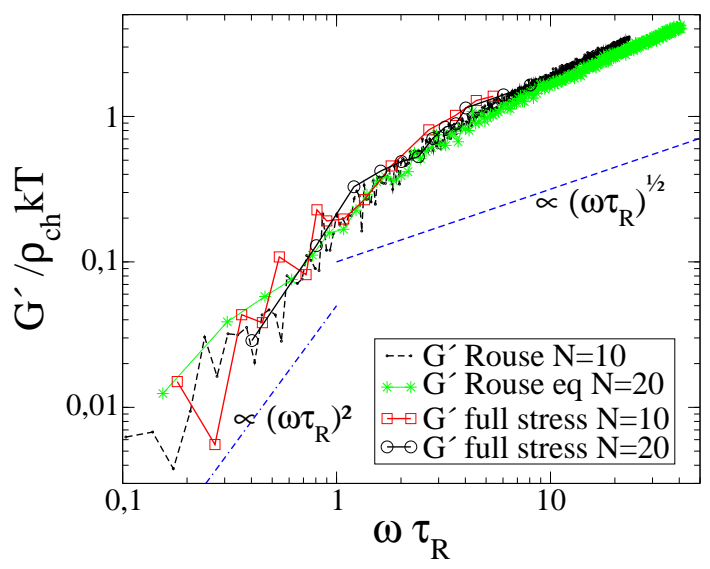

FIG. 10: Storage modulus divided by the chain density times $k_{B} T$ as a function of the reduced frequency $\omega \tau_{R}$ for the two systems. A cross over in the behavior is visible for $\omega=1 / \tau_{R}$.

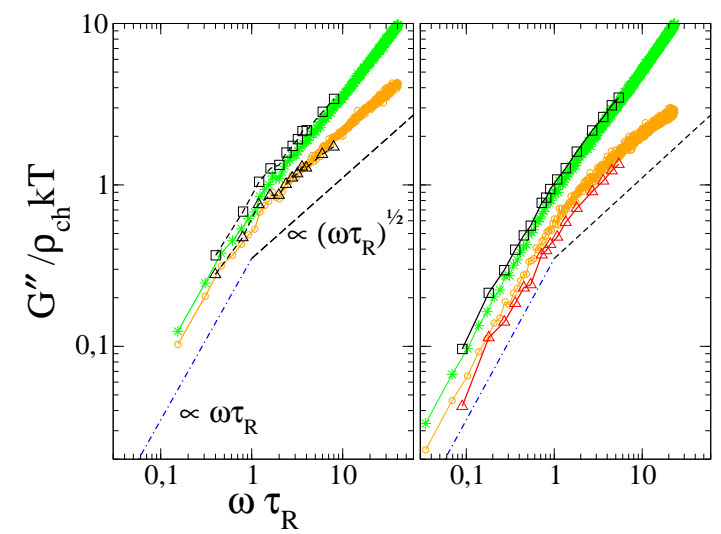

FIG. 11: Loss modulus divided by the chain density times $k_{B} T$ as a function of the reduced frequency $\omega \tau_{R}$ for the two systems $\left(N=20\right.$ - left, $N=10$ - right). NEMD full stress $G^{\prime \prime}$ squares, NEMD intra molecular stress $G^{\prime \prime}$ - triangles, corrected Rouse calculation - stars, Rouse calculation without short times correction - small circles. A crossover in the intra molecular component can be seen for $\omega=1 / \tau_{R}$. "Non polymer" relaxation mask the slope change for the full stress $G^{\prime \prime}(\omega)$.

Following the discussion of the linear properties of the melt we can further explore 
the non linear behavior studied by NEMD, considering the results presented in fig. 5 . Both the relative decrease in moduli and the harmonics intensity show that the storage modulus has a stronger non linear behavior compared to the loss modulus at a given shear rate. As we have shown that $G^{\prime}(\omega)$ has, at all considered frequencies, a large intra molecular contribution, and given that the bond potential is much steeper than the pair potential acting between all monomers, it is reasonable to expect that non linear effects due to large deformations would be more pronounced and would appear earlier for the storage modulus. From fig. higher the shear rate determining the onset of non-linear behavior. This critical value of the shear rate was found to vary linearly with frequency $\left(\gamma_{0} \omega\right)_{c} \propto \omega$. The system starts behaving as viscoelastic, and the linear regime extends to higher shear rates. In fact, as global chain relaxation does not take place on the time scale of the oscillations, there are already "frozen" slow vibration modes in the linear regime at low shear rates. Thus the relevant shear rate for the onset of non- linearities is shifted from $1 / \tau_{R}=1 / \tau_{1}$ to $1 / \tau_{p}$, where $p>1$ is the number of a higher vibration mode that still relaxes on the oscillations time scale at the given frequency.

Finally, we can summarize the overall mechanical behavior of the studied polymer melt exhibiting several distinct regimes as shown in fig. 12. At low frequencies $0 \leq \omega<1 / \tau_{R}$ and low shear rate amplitudes $\gamma_{0} \omega<1 / \tau_{R}$ the melt has Newtonian behavior. The viscosity is independent of shear rate and the elastic response is rather small as most of the stress is relaxed by the chain conformations. At low frequencies and high shear rates $\gamma_{0} \omega>1 / \tau_{R}$ the system is non linear: shear thinning in viscosity, softening in moduli and harmonics in the measured stress. When we shift to high frequencies $\omega>1 / \tau_{R}$ the melt exhibits more pronounced viscoelastic behavior with increasing storage modulus due to "frozen" modes and less intra molecular stress relaxation. The non linear boundary becomes frequency dependent and is shifted to higher shear rates determined by the relaxation time scale of chain segments of length $<N$. The melt is then expected to exhibit glassy behavior at very high frequencies when no subchain relaxation whatsoever can occur within an oscillation. 


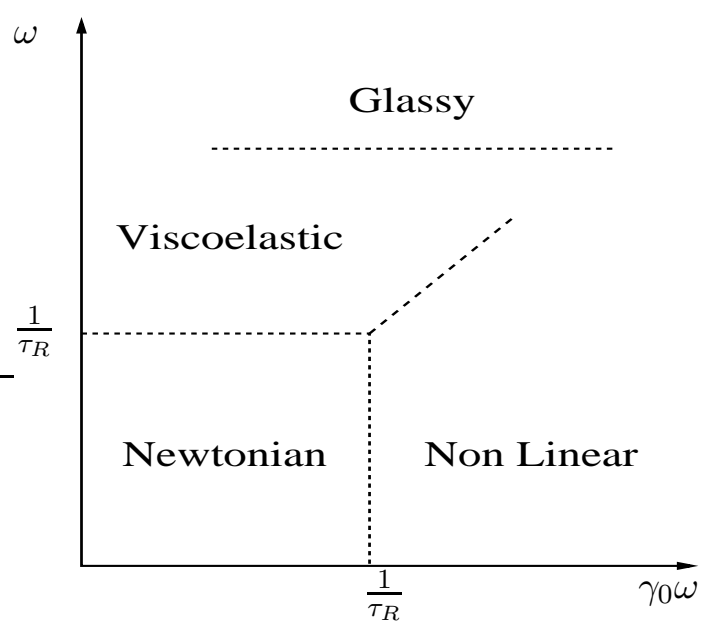

FIG. 12: Mechanical behavior of the melt for different frequencies and shear rates

\section{DISCUSSION AND CONCLUSIONS}

We have discussed the visco-elastic response of a model unentangled polymer melt to external shear strain. We use non equilibrium molecular dynamics methods to directly measure the elastic moduli and the viscosity of the melt. Our aim was also to investigate an equilibrium based method for these quantities, inspired by Green-Kubo relations and possibly offering greater precision. The method we proposed is inspired by the Rouse model, and based on a measurement of the vibration modes of the chains at equilibrium. This single chain quantity can be rather accurately measured from an equilibrium simulation. Following the philosophy of the Rouse model, we use the Rouse modes of the chains to estimate the long times mechanical behavior of the melt and the equilibrium stress correlation function to estimate small times, independent of chain length non-polymeric behavior. The resulting model provides a satisfactory description of the viscosity and of the elastic moduli of the melt. The part of the different contributions in the mechanical stress played in the visco-elastic melt behavior was also discussed. The NEMD results show that inter chain interactions in the melt add a non negligible background part to the storage modulus and dictate the loss modulus behavior at high frequencies. These interactions should be taken into account for a precise description of the mechanical properties of a polymer melt made of relatively short chains. More generally, these contributions 
are important for longer chains in several far from equilibrium situations including relaxation. We measured the chain Rouse modes mean square equilibrium values and mode correlations directly from our simulation, so that these quantities already contain, in a "mean field" way, some information about the background environment of the polymer chains. After completing this measurements quantitatively with short times estimate of the stress behavior, the obtained values for the mechanical properties of the melt are accurate, compared to non equilibrium "direct" measurements. The NEMD results show that the storage modulus depends on mode relaxation over a large frequency range, the energy storage takes place in chain conformations whereas for the loss modulus vibration modes are "frozen" one by one as the frequency grows higher than the inverse chain relaxation time and dissipation is dictated by short time scales "non polymer" stress relaxation. A given vibration mode $p$ relaxing over $\tau_{p}$, interpreted as the relaxation of a sub-chain of length $N / p$ monomers, can contribute mostly to the loss modulus $\left(\omega<1 / \tau_{p}\right)$ or to the storage modulus, if $\omega>1 / \tau_{p}$. This picture provides an explanation for the overall behavior of the elastic moduli in the studied frequency range. Our results are in quantitative agreement with previous studies, [8] but here we focused on lower frequencies for the elastic moduli, based on the chain relaxation time that we estimated, in order to interpret the microscopic mechanisms involved. Different stress contributions on different time scales were measured from the simulations, thus allowing the determination of mechanical response from equilibrium properties involving Rouse modes measurements and short times corrections.

We examined the onset of non linear effects in the measured quantities, manifested by shear thinning, moduli softening and harmonics in the stress time series. The non linear regime is dictated by the shear rate of the solicitation and takes place at $\gamma_{0} \omega>1 / \tau_{R}$ for $\omega<1 / \tau_{R}$. At higher frequencies, onset of nonlinear effects is related to the strain amplitude rather than rate, as full chain relaxation does not take place on the time scale of the oscillations. Our study allows a comprehensive description of the melt mechanical behavior in the form of a schematic frequency - shear rate diagram shown in fig. 12 .

We finally mention that the general method presented here is not, in principle, limited to unentangled melts. Indeed, in the general reptation picture, the formula used for the 
stress tensor is the same as that used in the Rouse model, formula 13. The relaxation of the modes will be dramatically slowed down by entanglement effects, so that the viscosity will increase rapidly. Prefactors, however, are associated with equilibrium correlation functions and are not affected by entanglement effects.

[1] K. Kremer, G. S. Grest,J. Chem. Phys. 1990, 92, 5057.

[2] J. D. Ferry, "Viscoelastic Properties of Polymers", $3^{\text {rd }}$ edition, Wiley, New York 1980.

[3] R. G. Larson, "Constitutive Equations for Polymer Melts and Solutions", Butterworth Publ., AT\&T Bell Labs 1988.

[4] M. Doi, S. F. Edwards, "The Theory of Polymer Dynamics", Oxford University Press, 1986.

[5] M. Kröger,Phys. Rep. 2004, 390, 453.

[6] M. Kröger, S. Hess, Phys. Rev. Lett 2000, 85, 1128.

[7] M. Kröger, C. Luap, R. Muller,Macromolecules 1997, 30, 526.

[8] J. G. H. Cifre, S. Hess, M. Kröger,Macromol. Theory Simul. 2004, $13,748$.

[9] D. J. Evans, G. P. Morris, "Stat Mechanics of Noneq Liq", Academic, New York, 1990.

[10] M. P. Allen, D. J. Tildesley, "Computer Simulation of Liquids", Oxford University Press, 1987.

[11] P. Keblinski, private communication. S. Sen, S.K. Kumar and P. Keblinski, Preprint 2006.

[12] Z. Xu, R. Khare, J. J. de Pablo, S. Kim,J. Chem. Phys. 1997, 106, 8285.

[13] C. Bennemann, J. Baschnagel, W. Paul, K. Binder, Comp. and Theor. Polym. Sc. 1999, 9, 217.

[14] V. A. Harmandaris, V. G. Mavrantzas, D. N. Theodorou, Macromolecules 1998, 31, 7934.

[15] S. Sen, S. K. Kumar, P. Keblinski, Macromolecules 2005, 38, 650.

[16] M. Mondello, G. S. Grest,J. Chem. Phys. 1997, 106, 9327.

[17] S. J. Plimpton, J. Comp. Phys. 1995, 117, 1.

LAMMPS web site: www.cs.sandia.gov $/{ }^{\sim}$ sjplimp/lammps.html 